\title{
A crucial role of angiotensin converting enzyme 2 (ACE2) in SARS coronavirus-induced lung injury
}

\author{
Keiji Kuba ${ }^{1,7}$, Yumiko Imai ${ }^{1,7}$, Shuan Rao $^{2,7}$, Hong Gao ${ }^{3}$, Feng Guo ${ }^{2}$, Bin Guan ${ }^{2}$, Yi Huan ${ }^{2}$, Peng Yang ${ }^{2}$, \\ Yanli Zhang ${ }^{2}$, Wei Deng ${ }^{3}$, Linlin Bao ${ }^{3}$, Binlin Zhang ${ }^{3}$, Guang Liu ${ }^{2}$, Zhong Wang ${ }^{4}$, Mark Chappell ${ }^{5}$, \\ Yanxin Liu ${ }^{2}$, Dexian Zheng ${ }^{2}$, Andreas Leibbrandt ${ }^{1}$, Teiji Wada ${ }^{1}$, Arthur S Slutsky $^{6}$, Depei Liu ${ }^{2}$, Chuan Qin ${ }^{3}$, \\ Chengyu Jiang $^{2} \&$ Josef M Penninger ${ }^{1}$
}

During several months of 2003, a newly identified illness termed severe acute respiratory syndrome (SARS) spread rapidly through the world ${ }^{1-3}$. A new coronavirus (SARS-CoV) was identified as the SARS pathogen ${ }^{4-7}$ which triggered severe pneumonia and acute, often lethal, lung failure ${ }^{8}$. Moreover, among infected individuals influenza such as the Spanish flu 9,10 and the emergence of new respiratory disease viruses ${ }^{11,12}$ have caused high lethality resulting from acute lung failure ${ }^{13}$. In cell lines, angiotensin-converting enzyme 2 (ACE2) has been identified as a potential SARS$\mathrm{CoV}$ receptor ${ }^{14}$. The high lethality of SARS-CoV infections, its enormous economic and social impact, fears of renewed outbreaks as well as the potential misuse of such viruses as biologic weapons make it paramount to understand the pathogenesis of SARS-CoV. Here we provide the first genetic proof that ACE2 is a crucial SARS-CoV receptor in vivo. SARS-CoV infections and the Spike protein of the SARS-CoV reduce ACE2 expression. Notably, injection of SARS-CoV Spike into mice worsens acute lung failure in vivo that can be attenuated by blocking the renin-angiotensin pathway. These results provide a molecular explanation why SARS-CoV infections cause severe and often lethal lung failure and suggest a rational therapy for SARS and possibly other respiratory disease viruses.

Recently, ACE2 was identified as a functional SARS coronavirus receptor in cell lines ${ }^{14}$. But a possible second receptor, CD209L (L-SIGN), has also been identified from in vitro studies ${ }^{15}$. Thus, it was not known whether ACE2 is indeed crucial for SARS-CoV infections in vivo. To address this question genetically, we infected Ace 2 knockout ${ }^{16}$ and control wild-type mice with SARS-CoV. As reported previously ${ }^{17}$, SARS$\mathrm{CoV}$ infections of wild-type mice result in viral replication in the lungs and the recovery of large amounts $\left(>10^{7}\right.$ the tissue culture-infectious dose, which will infect $50 \%$ of the cell monolayers $\left(\mathrm{TCID}_{50} \mathrm{~T}\right)$ per gram lung tissue) of infectious virus (Fig. 1a). In Ace 2 knockout mice, only a very low quantity of infectious SARS-CoV virus could be recovered $\left(<10^{2}\right.$ TCID $_{50}$ per gram lung tissue; Fig. 1a), and the copy numbers of SARS-CoV Spike RNA were greatly reduced (Fig. 1b). SARS-CoV infection of mice is associated with the development of mild pathological changes in the lungs (Fig. 1c,d). Moreover, pathologic alterations in lungs were reduced in Ace 2 mutant mice compared to wild-type mice (Fig. 1c,d). These data provide the first genetic proof that ACE2 is indeed a crucial in vivo SARS receptor required for effective replication of infectious SARS-CoV.

We have recently shown that the renin-angiotensin system has a crucial role in severe acute lung injury and that the SARS-CoV receptor ACE2 has a protective role in acute lung failure ${ }^{18}$ (Fig. 2a). Notably, experimental SARS-CoV infections of wild-type mice in vivo resulted in considerably reduced ACE2 expression in the lungs (Fig. 2b) suggesting that reduced ACE2 expression might have a role in SARS-CoV-mediated severe acute lung pathologies. By contrast, ACE lung expression levels were not overtly changed in SARS-CoV-infected mice (Fig. 2b). We therefore speculated that SARS-CoV might affect lung pathologies through ACE2. To test this idea, we established a defined model system using recombinant SARS-CoV surface-Spike protein, which is the essential ligand for ACE2 binding ${ }^{14}$. This model system allowed us to avoid possible secondary effects resulting from viral replication or infections in vivo and to directly test whether SARS-CoV Spike protein might adversely affect acute lung injury through modulation of ACE2.

We first tested whether recombinant SARS-CoV Spike protein (Supplementary Fig. 1 online) binds to human as well as mouse ACE2 protein using in vitro pull-down assays. Our recombinant Spike-Fc protein indeed pulled down both human and mouse ACE2 (Fig. 2c). SARS-CoV Spike-Fc binding to human and mouse ACE2 was confirmed by FACS binding assays of Spike-Fc to 293 cells overexpressing human or mouse ACE2 (Fig. 2d). Moreover, Spike-Fc bound to endogenous

\footnotetext{
${ }^{1}$ Institute of Molecular Biotechnology of the Austrian Academy of Sciences, Dr. Bohr-gasse 7, A-1030 Vienna, Austria. ${ }^{2}$ National Laboratory of Medical Molecular Biology, Institute of Basic Medical Sciences, ${ }^{3}$ Institute of Laboratory Animal Sciences and ${ }^{4}$ Peking Union Medical College Hospital, Chinese Academy of Medical Sciences \& Peking Union Medical College, 5 Dongdan Santiao, Beijing 100005, China. ${ }^{5}$ The Hypertension and Vascular Disease Center, Wake Forest University School of Medicine, Gray Building, G054 Medical Center Boulevard, Winston-Salem, North Carolina 27157-1032, USA. ${ }^{6}$ Department of Medicine and Interdepartmental Division of Critical Care, University of Toronto; St. Michael's Hospital, 30 Bond Street, Toronto, M5B 1W8, Canada. ${ }^{7}$ These authors contributed equally to this work. Correspondence should be addressed to C.J. (jiang@pumc.edu.cn).
} 


\section{LETTERS}

a

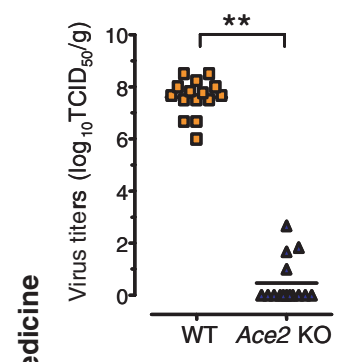

b

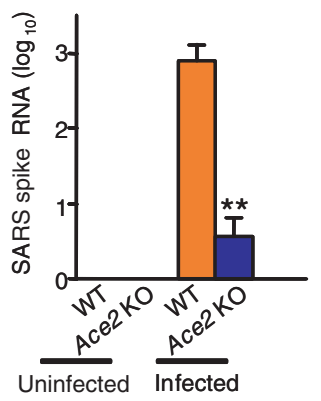

C

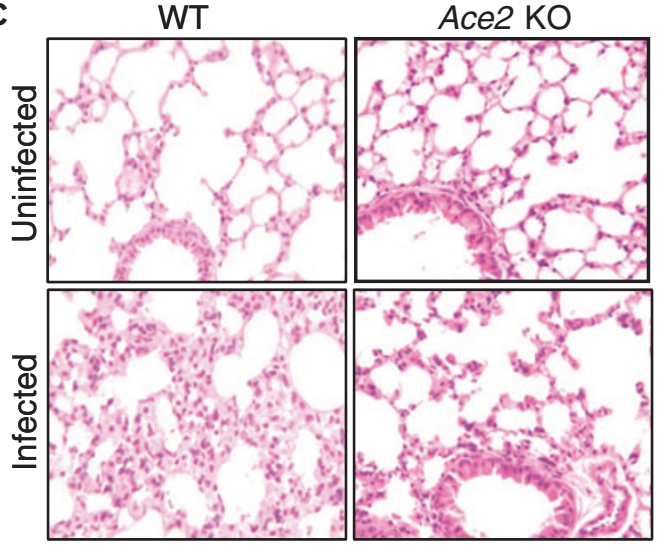

d

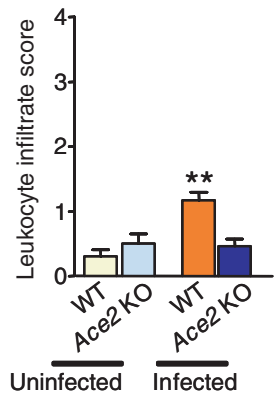

Figure 1 ACE2 is a crucial receptor for SARS-CoV infections in vivo. (a,b) SARS-CoV replication (a) and detection of SARS-CoV Spike RNA (b) in wild-type (WT) and Ace2 knockout mice. Viral replication was determined from lung tissue at day 2 of infection. Virus titers (mean $\log _{10} T_{C I D} D_{50}$ per gram lung tissue) are shown for individual mice. $n=15$ per group. SARS-CoV Spike RNA expression was assayed using real-time RT-PCR and normalized to mouse Actb. Data are shown as mean + s.e.m. $n=15$ per group. ${ }^{*} P<0.01$. (c) Lung histopathology (original magnification $\times 200$ ) and (d) lung injury scores as defined by leukocyte infiltration of control and SARS-CoV-infected wild-type and Ace2 knockout mice. Lung samples were taken on day 6 after SARS-CoV infection.

ACE2 in Vero E6 cells (Fig. 2e). Notably, binding of Spike-Fc to endogenous ACE2 in Vero E6 cells resulted in downregulation of ACE2 surface expression (Fig. 2e and Supplementary Fig. 1 online). Spike-Fc also decreased surface levels of human and mouse ACE2 overexpressed in 293 cells (data not shown) and triggered syncytia formation of mouse ACE2-transfected but not control CD4-transfected 293 cells (not shown). Thus, analogous to other virus-receptor interactions ${ }^{19}$, SARS-
CoV Spike protein binding to ACE2 in cell lines or SARS-CoV infections in vivo results in reduced ACE2 protein expression.

Because ACE2 is a crucial SARS-CoV receptor (Fig. 1), SARS-CoV Spike protein binding to ACE2 downmodulates ACE2 expression (Fig. 2), and loss of ACE2 expression results in severe acute respiratory failure $^{18}$, we tested whether SARS-CoV Spike protein, which is the crucial ACE2 binding protein ${ }^{20,21}$, could affect the severity of acute lung injury in a

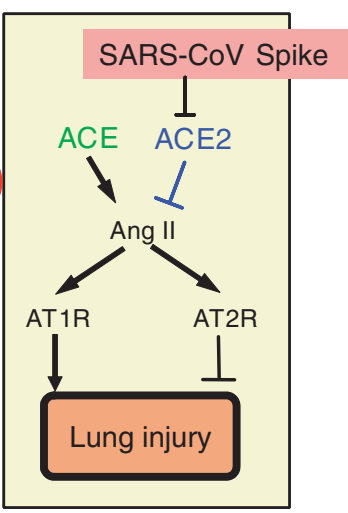

b

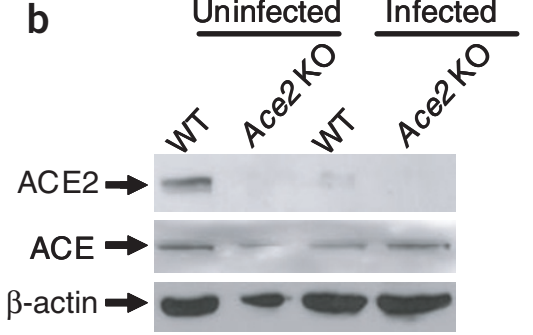

C

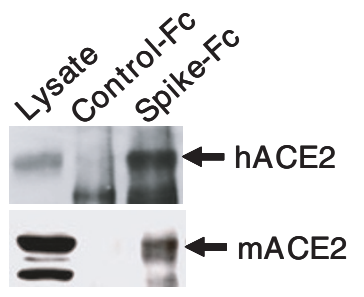

d
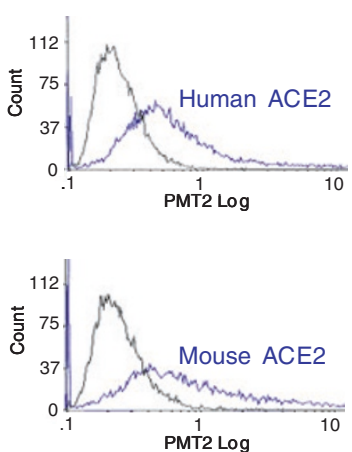

e
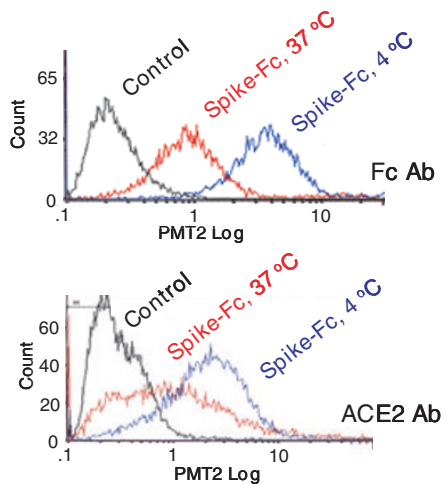

Figure 2 Downregulation of ACE2 expression by SARS-CoV infection and SARS-CoV Spike protein. (a) Schematic diagram of the renin-angiotensin system in acute lung failure and proposed SARS-CoV action. (b) Decreased ACE2 protein, but normal ACE levels, in the lungs of SARS-CoV-infected mice. Lung homogenates were prepared from control and SARS-CoV-infected wild-type or Ace2 knockout (KO) mice on day 2 and analyzed by western blot. (c) Binding of recombinant Spike(S-1190)-Fc protein to human ACE2 (hACE2) and mouse ACE2 (mACE2) in pull-down assays. Spike-Fc but not controlFc protein pulled down hACE2 and mACE2 from total-cell extracts of A549 human alveolar epithelial cells and IMCD mouse kidney epithelial cells, respectively. Total lysates are shown as controls. (d) Binding of Spike-Fc protein to human and mouse ACE2 in cell culture. 293 cells transfected with hACE2 or mACE2 were incubated with Spike-Fc and the binding was detected by FACS (blue lines). Nontransfected 293 cells incubated with Spike-Fc followed by Fc-specific antibodies are shown as controls (black line). (e) Decreased cell-surface expression of ACE2 after binding to Spike-Fc protein at $37^{\circ} \mathrm{C}$ compared to $4^{\circ} \mathrm{C}$ in Vero E6 cells. ACE2 surface expression was detected at $3 \mathrm{~h}$ of incubation with Spike-Fc using an ACE2-specific monoclonal antibody. Similar data were obtained using Fc-specific antibody to directly detect surface-bound Spike-Fc and to avoid masking of the ACE2 epitope. Representative FACS histograms are shown including a background control with an isotype-matched antibody. 
a

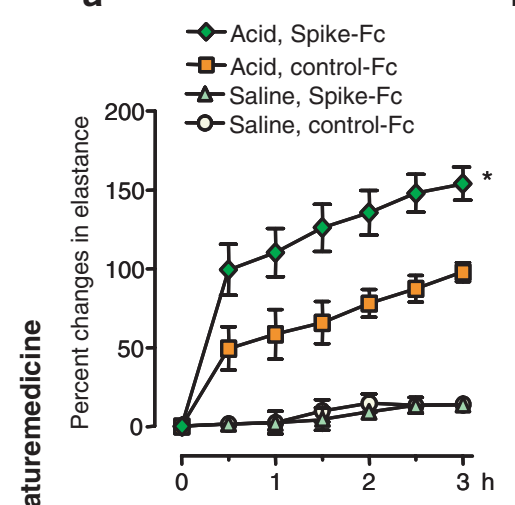

b

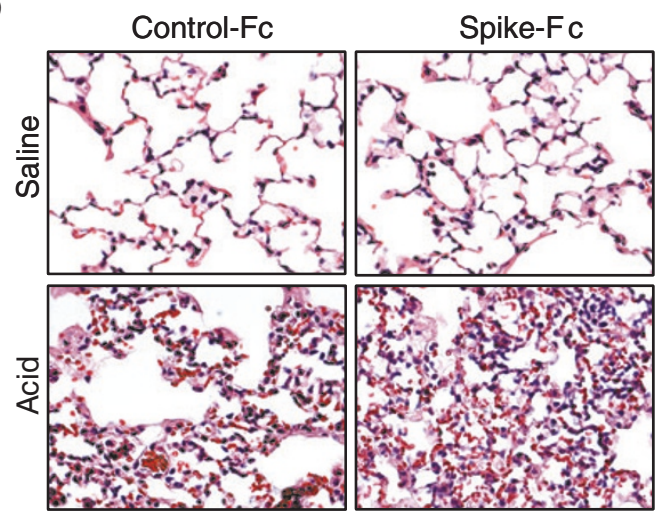

C

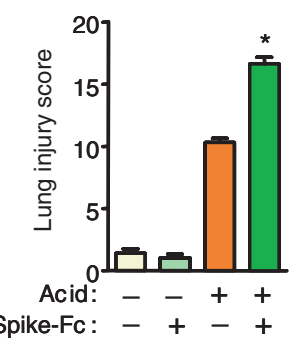

d

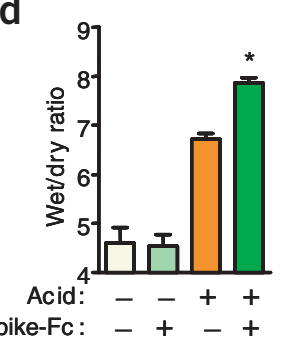

f

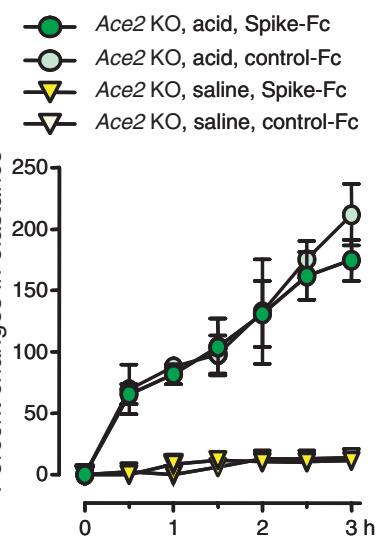

Figure 3 The SARS-CoV Spike protein enhances the severity of acute lung injury. (a) Lung elastance measurements after saline or acid instillation in Spike-Fc protein- $(5.5 \mathrm{nmol} / \mathrm{kg})$ or control-Fc(5.5 nmol $/ \mathrm{kg}$ ) treated wild-type mice. $n=5-7$ per group. ${ }^{*} P<0.05$ for the whole time course comparing Spike-Fc-treated and control-Fctreated wild-type mice after acid injury. (b) Lung histopathology. Representative images are shown. Original magnification, $\times 200$. (c) Lung injury score (Supplementary Table 1 online). ${ }^{*} P<0.01$ versus control-Fc-treated wild-type. (d) Wet to dry weight ratios of lungs as readout for pulmonary edema in control and Spike-Fc-treated mice in the presence or absence of acid-induced lung injury.

${ }^{*} P<0.05$ between control and Spike-Fc-treated mice with acid challenge. (e) Severe acute lung failure by Spike(S318-510)-Fc ( $5.5 \mathrm{nmol} / \mathrm{kg})$ treatment in acid-challenged mice. The scheme (upper panel) shows the ACE2-binding domain of Spike (S318-510). Lung elastance measurements (lower panel) showed that Spike(S318-510)Fc induced severe acute lung failure in acidchallenged wild-type mice, comparable to Spike(S1190)-Fc. $n=5-7$ per group. $P<0.05$ for the whole time course comparing Spike(S318510)-Fc or Spike(S1190)-Fc-treated and controlFc-treated wild-type mice after acid injury. (f) Lung elastance measurements after acid instillation in Spike-Fc protein-(S1190; $5.5 \mathrm{nmol} / \mathrm{kg}$ ) or controlFc- $(5.5 \mathrm{nmol} / \mathrm{kg}$ ) treated Ace2 knockout (KO) mice. $n=5-7$ for each group.

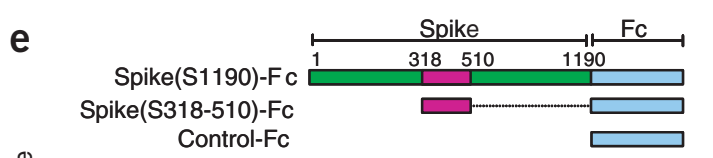
Control-Fc

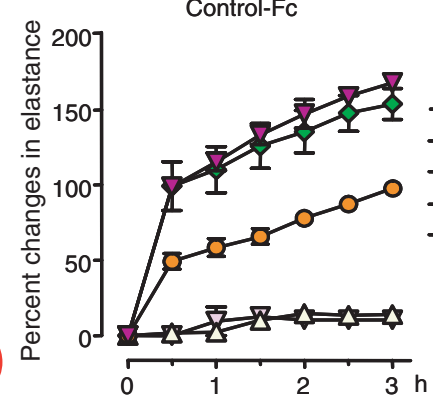

$\rightarrow$ Acid, Spike (S318-510)-Fc $\checkmark$ Acid, Spike (S1190)-Fc - Acid, control-Fc $-\nabla$ - Saline, Spike (S318-510)-Fc $\triangle$ Saline, control-Fc vivo. Notably, treatment with Spike-Fc protein worsened the lung function in wild-type mice, whereas control-Fc protein showed no apparent effects (Fig. 3a). Moreover, Spike-Fc treatment of acid-challenged wild-type mice augmented the pathological changes in the lung parenchyma (Fig. 3 b,c and Supplementary Table 1 online) and increased lung edemas as defined by a wet/dry lung weight ratios (Fig. 3d). We next made a Spike-deletion mutant that only contained the previously mapped ACE2-binding domain (amino acids 318-510) ${ }^{21}$ fused to human Fc (Fig. 3e and Supplementary Fig. 1 online). This short Spike(S318-510)-Fc protein containing the minimal ACE2 binding site also binds to ACE2 in cell lines using FACS assays and downmodulates the cell-surface expression of ACE2 (Supplementary Fig. 1 online). Treatment with Spike(S318510)-Fc again worsened acid-induced acute lung injury in wild-type mice (Fig. 3e). Notably, in vivo Spike-Fc protein administration did not affect the severity of lung failure in Ace 2 knockout mice (Fig. 3f), indicating that the effect of Spike protein on acute lung injury is ACE2 specific.

To further clarify whether the intraperitoneally injected Spike-Fc protein directly affected lung pathology in mice, we examined the localization of the injected Spike-Fc protein in lung. Spike-Fc was detected in lung homogenates by western blot using human Fc-specific antibody (Fig. 4a), whereas injected control-Fc was not detected. In addition, using immunohistochemistry, we found that Spike-Fc protein localized to bronchial epithelial cells, inflammatory exudates and alveolar pneumocytes (Fig. 4b). Notably, Spike-Fc primarily localized to severe lesions (Fig. 4b). This localization of Spike-Fc protein is similar to Spike antigen staining in SARS-CoV-infected mice ${ }^{22}$. Spike-Fc treatment resulted in downregulation of ACE2 protein expression in lungs of acid-treated wild-type mice in vivo (Fig. 4c), consistent with ACE2 protein downregulation in SARS-CoV-infected mice (Fig. 2a) and Spike-Fc proteintreated cells in vitro (Fig. 2e). These results show that the SARS-CoV Spike protein can directly affect the development of severe acute lung failure through ACE2.

ACE2 functions as a carboxypeptidase, cleaving a single residue from angiotensin I (AngI), generating Ang1-9 (refs. 23,24), and a single residue from angiotensin II (AngII) to generate Ang1-7 (ref. 23). The ACE2 homologue ACE, by contrast, cleaves the decapeptide AngI into the octapeptide AngII ${ }^{25}$. Thus, ACE2 counterbalances the function of ACE and negatively regulates AngII production (Fig. 2a). To test whether Spike-Fc injections indeed affect the function of the renin-angiotensin system, we analyzed AngII levels in the lungs of acid- and Spike-Fctreated mice. Acid aspiration increased AngII levels in the lungs of wild-type mice. Notably, we observed a further, significant increase in AngII levels in the lung tissue of mice treated with Spike-Fc (Fig. 4d). To confirm whether Spike-Fc promotes lung disease pathogenesis through increased AngII production and functional alterations of the reninangiotensin system, we blocked the AngII receptor type 1 (AT1R) ${ }^{26}$ with a specific inhibitor. The AT1R is the crucial receptor that mediates AngII-induced vascular permeability and severe acute lung injury ${ }^{18}$. 


\section{LETTERS}

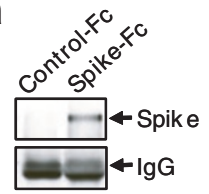

C

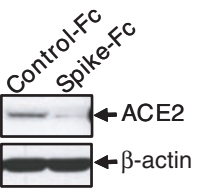

d

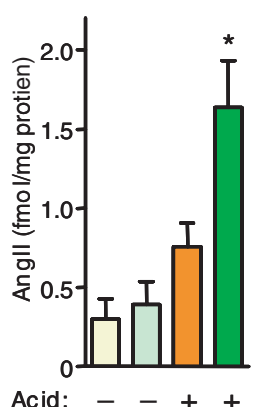

Spike-Fc: -+-+ b

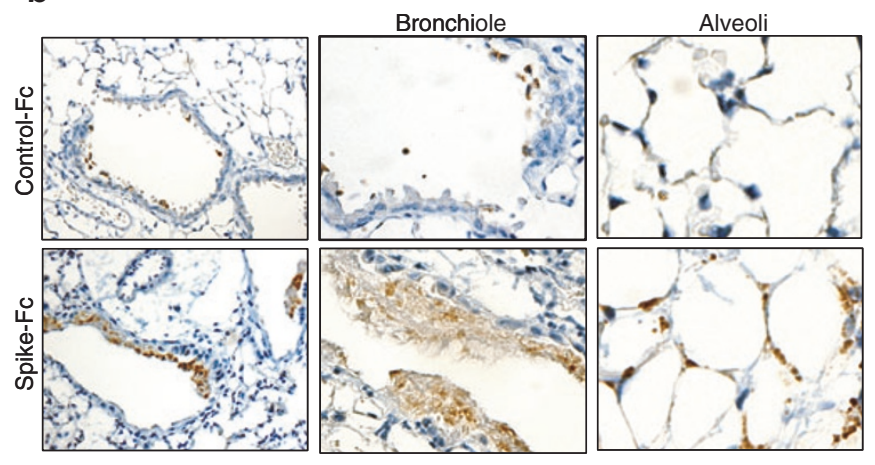

e

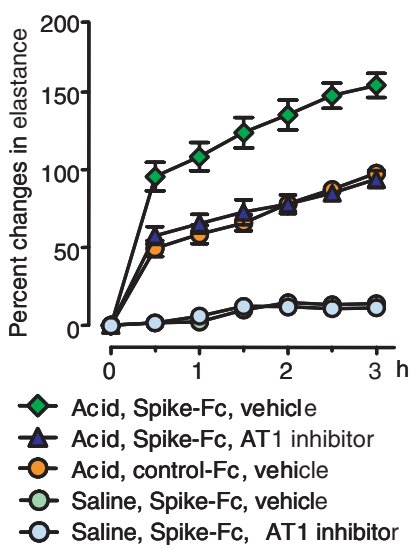

f

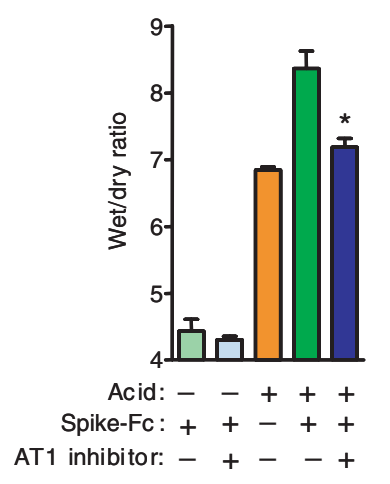

Figure 4 SARS-CoV Spike mediates lung injury through modulation of the renin-angiotensin system. (a,b) Localization of intraperitoneally injected Spike(S-1190)-Fc in lung tissue. (a) Spike-Fc was detected by pull-down assay with Protein G Sepharose and western blot with human Fc-specific antibody. Mouse IgG is shown as loading control. (b) Lung immunohistochemistry to detect Spike(S-1190)-Fc or control-Fc protein using a human Fc-specific antibody. Spike(S1190)-Fc localizes to bronchial epithelial cells (left; original magnification, $\times 100$ ), inflammatory exudates cells (middle; original magnification, $\times 200$ ), and alveolar pneumocytes (right; original magnification, $\times 200$ ). (c) Decreased ACE2 protein expression in the lungs of Spike(S-1190)Fc- treated mice. Lung homogenates were prepared from control-Fc- and Spike(S-1190)-Fc-treated wild-type mice and analyzed by western blot with ACE2-specific antibody. (d) Angll peptide levels in lungs of Spike(S1190)-Fc protein- or control-Fctreated wild-type mice after saline or acid aspiration. Angll levels were determined at $3 \mathrm{~h}$ by enzyme immunoassay. Bars, mean \pm s.e.m. ${ }^{*} P<0.05$ comparing Spike(S1190)-Fc- and control-Fc-treated wild-type mice after acid injury. (e) Lung elastance measurements in acid plus Spike(S1190)-Fc-challenged wild-type mice treated with the AT1R inhibitor losartan $(15 \mathrm{mg} / \mathrm{kg})$. $n=4-6$ per group. $P<0.05$ comparing losartantreated Spike(S1190)-Fc-challenged mice with vehicle-treated Spike(S1190)-Fc-challenged mice. (f) Wet to dry weight ratios of lungs of acid and Spike(S1190)-challenged mice in the presence or absence of losartan (15 mg/kg). $n=4-6$ mice per group. ${ }^{*} P<0.05$, comparing losartan-treated wild-type with vehicle-treated wild-type mice at $3 \mathrm{~h}$ after acid injury.
Inhibition of the AT1R indeed attenuated acute severe lung injury in Spike-Fc-treated mice (Fig. 4e). Inhibition of the AT1R also attenuated pulmonary edema (Fig. 4f). Taken together, our data show that SARSCoV Spike can exaggerate acute lung failure through deregulation of the renin-angiotensin system. Moreover, SARS-CoV Spike-mediated lung failure can be rescued by inhibition of AT1R.

It has been estimated that the Spanish flu virus that killed more than 20 million people at the beginning of the twentieth century was lethal to around $0.5 \%$ of infected people ${ }^{9,10}$, whereas the lethality of SARS-CoV infections reached $10 \%$ even with modern intensive care treatment ${ }^{1-3}$. Given this very high lethality of SARS and the enormous economic and social impact of the worldwide SARS outbreak, elucidation of the disease pathogenesis is crucial for future treatment in case of renewed outbreaks. Moreover, a recent outbreak of avian influenza A (H5N1) in humans resulted in up to $70 \%$ lethality from acute respiratory failure ${ }^{12}$. Before the discovery of SARS-CoV, two coronaviruses (HCoV-229E and HCoVOC43) were known to infect humans, but they caused only self-limiting upper respiratory tract infections (30\% of the common colds) and had never been reported to cause severe illness ${ }^{27}$. The molecular determinants that may account for the dramatic differences in pathogenesis between these human coronaviruses and SARS-CoV were unknown.

Our data provide a molecular explanation for the severe lung failure and lethality associated with SARS: we hypothesize that infections with SARS-CoV result in ACE2 downregulation through binding of SARS$\mathrm{CoV}$ Spike protein to ACE2. Given that ACE2 is a key negative regulatory factor for severity of lung edema and acute lung failure, SARS-CoV Spike protein-mediated ACE2 downregulation then contributes to the severity of lung pathologies. This scenario would explain how this family member of the 'relatively harmless' coronaviruses has turned into a lethal virus. Notably, recent data in a small cohort of individuals with SARS suggested that an insertion deletion ACE polymorphism that affects ACE function correlates with disease severity ${ }^{28}$, implying that our findings are indeed relevant for humans.

Our data provide a molecular link between SARS pathogenesis and the role of the renin-angiotensin system in lung failure. Recombinant ACE2 protein therefore could not only be a treatment to block spreading of SARS-CoV, but modulation of the renin-angiotensin system could also be used to protect individuals with SARS, and possibly individuals infected with other viruses such as avian influenza A strains, from developing acute severe lung failure and acute respiratory distress syndrome.

\section{METHODS}

For details of Methods, see Supplementary Methods online.

In vivo SARS infections. The SARS-CoV (Beijing strain, $\mathrm{PUMC01}$ isolate) used in this study was provided by Z. Wang and Y. Liu (Chinese Academy of Medical Sciences, Chinese National Human Genome Center) ${ }^{29}$. All mouse studies were approved by the Ministry of Health Science and Technology division of the People's Republic of China. We intranasally inoculated mice with $100 \mu$ l virus $\left(10^{5.23} \mathrm{TCID}_{50}\right)$. At day 2 , mice were killed, and the lungs were removed for further analyses. We assessed lung injury scores as described previously ${ }^{30}$.

SARS-CoV Spike protein binding experiments. We cloned the coding sequence of SARS-CoV Spike protein (amino acids 1-1,190 from Urbani strain) or a Spike sequence that only contains the previously mapped ${ }^{21}$ ACE2-binding domain (amino acids 318-510) to generate a fusion protein with the Fc portion 
of human IgG1. We purified Spike-Fc protein from transfected CHO cells using affinity chromatography. For in vitro binding assays, we used cell lysates from A549 human alveolar epithelial cells or IMCD mouse kidney epithelial cells, and we pulled down Spike-Fc or control human IgG-Fc protein using Protein G Sepharose followed by western blot. For flow cytometry, we detached Vero E6 cells using a $2 \mathrm{mM}$ mixture of EDTA and PBS and incubated them with Spike-Fc or control human IgG-Fc protein at $4{ }^{\circ} \mathrm{C}$ or $37^{\circ} \mathrm{C}$ for $3 \mathrm{~h}$. We then incubated cells with ACE2-specific antibodies or a FITC-conjugated human IgG-specific antibody.

Recombinant Spike-Fc in vivo challenge in mice. We used the mouse model of acid aspiration-induced acute lung injury ${ }^{18}$ for all Spike-Fc in vivo experiments. Mice received Spike(S1190)-Fc, Spike(S318-510)-Fc or control-Fc $(5.5 \mathrm{nmol} / \mathrm{kg}$ each) intraperitoneally three times (at 30 min before and at 1 and $2 \mathrm{~h}$ after) during acid treatment. For AT1 inhibition of Spike-Fc-mediated acute lung injury, we treated the Spike(S1190)-Fc-challenged mice with the AT1 inhibitor losartan $(15 \mathrm{mg} / \mathrm{kg})$. Lung injury scores were assessed as described previously ${ }^{30}$.

Statistical analyses. All data are shown as mean \pm s.e.m. Measurements at single time points were analyzed by unpaired $t$-test (virus titers, SARS-CoV Spike-RNA levels), ANOVA with two tailed $t$-test (percent changes in elastance, wet/dry ratio AngII levels) or Kruskal-Wallis test (histological scores). Time courses were analyzed by repeated measurements (mixed model) ANOVA with Bonferroni post $t$ tests. All statistical tests were calculated using the GraphPad Prism 4.00 (GraphPad Software) and a JMP (SAS Institute) programs. $P<0.05$ was considered to indicate statistical significance.

Accession number. The Genbank accession number for the SARS coronavirus (Beijing strain, PUMC01 isolate) is AY350750.

Note: Supplementary information is available on the Nature Medicine website.

\section{ACKNOWLEDGMENTS}

We thank C. Richardson and all members of our laboratory for discussions. We also thank B. Seed for discussion and providing the systems to generate recombinant Spike proteins. We thank Q. Zhu, L. Ruan and L. Zhang for sharing unpublished data of SARS coronavirus infection on mice and protocols of virus infections. This work is supported by the Institute for Molecular Biotechnology of the Austrian Academy of Sciences and the Jubilaeumsfonds of the Austrian National Bank. K.K. is supported by a Marie Curie Fellowship from the EU.

(2) C.J. is supported by Beijing Committee of Science and Technology grant H030230010930, National Natural Science Foundation of China innovation group grant 30421003 and SARS donation from Joincare Corporation. A.S. is supported in part by the Canadian Institutes of Health Research and the Canada Foundation for Innovation.

\section{COMPETING INTERESTS STATEMENT}

The authors declare competing financial interests (see the Nature Medicine website for details).

Received 22 April; accepted 3 June 2005

Published online at http://www.nature.com/naturemedicine/

1. Tsang, K.W. et al. A cluster of cases of severe acute respiratory syndrome in Hong Kong. N. Engl. J. Med. 348, 1977-1985 (2003).

2. Lee, N. et al. A major outbreak of severe acute respiratory syndrome in Hong Kong. N. Engl. J. Med. 348, 1986-1994 (2003).
3. Poutanen, S.M. et al. Identification of severe acute respiratory syndrome in Canada. N. Engl. J. Med. 348, 1995-2005 (2003).

4. Ksiazek, T.G. et al. A novel coronavirus associated with severe acute respiratory syndrome. N. Engl. J. Med. 348, 1953-1966 (2003).

5. Drosten, C. et al. Identification of a novel coronavirus in patients with severe acute respiratory syndrome. N. Engl. J. Med. 348, 1967-1976 (2003).

6. Rota, P.A. et al. Characterization of a novel coronavirus associated with severe acute respiratory syndrome. Science 300, 1394-1399 (2003).

7. Marra, M.A. et al. The Genome sequence of the SARS-associated coronavirus. Science 300, 1399-1404 (2003).

8. Severe Acute Respiratory Syndrome (SARS) Epidemiology Working Group. WHO consensus document on the epidemiology of severe acute respiratory syndrome (SARS). (http://www.who.int/csr/sars/en/WHOconsensus.pdf, 2003).

9. Oxford, J.S. Influenza A pandemics of the 20th century with special reference to 1918: virology, pathology and epidemiology. Rev. Med. Virol. 10, 119-133 (2000).

10. Johnson, N.P. \& Mueller, J. Updating the accounts: global mortality of the 19181920 "Spanish" influenza pandemic. Bull. Hist. Med. 76, 105-115 (2002).

11. Tran, T.H. et al. Avian influenza A (H5N1) in 10 patients in Vietnam. N. Engl. J. Med. 350, 1179-1188 (2004).

12. World Health Organization. Confirmed human cases of avian influenza A (H5N1), 7 September 2004. (http://www.who.int/csr/disease/avian_influenza/country/cases table_2004_09_07/en/print.html, 2004).

13. Fouchier, R.A. et al. Avian influenza A virus (H7N7) associated with human conjunctivitis and a fatal case of acute respiratory distress syndrome. Proc. Natl. Acad. Sci. USA 101, 1356-1361 (2004).

14. Li, W. et al. Angiotensin-converting enzyme 2 is a functional receptor for the SARS coronavirus. Nature 426, 450-454 (2003).

15. Jeffers, S.A. et al. CD209L (L-SIGN) is a receptor for severe acute respiratory syndrome coronavirus. Proc. Natl. Acad. Sci. USA 20, 20 (2004).

16. Crackower, M.A. et al. Angiotensin-converting enzyme 2 is an essential regulator of heart function. Nature 417, 822-828 (2002).

17. Subbarao, K. et al. Prior infection and passive transfer of neutralizing antibody prevent replication of severe acute respiratory syndrome coronavirus in the respiratory tract of mice. J. Virol. 78, 3572-3577 (2004).

18. Imai, Y. et al. The SARS-coronavirus receptor angiotensin coverting enzyme 2 protects from severe acute lung failure. Nature (in the press).

19. Pelchen-Matthews, A., Signoret, N., Klasse, P.J., Fraile-Ramos, A. \& Marsh, M. Chemokine receptor trafficking and viral replication. Immunol. Rev. 168, 33-49 (1999).

20. Yang, Z.Y. et al. pH-dependent entry of severe acute respiratory syndrome coronavirus is mediated by the spike glycoprotein and enhanced by dendritic cell transfer through DC-SIGN. J. Virol. 78, 5642-5650 (2004).

21. Wong, S.K., Li, W., Moore, M.J., Choe, H. \& Farzan, M.A 193-amino acid fragment of the SARS coronavirus S protein efficiently binds angiotensin-converting enzyme 2. J. Biol. Chem. 279, 3197-3201 (2004).

22. Roberts, A. et al. Aged BALB/c mice as a model for increased severity of severe acute respiratory syndrome in elderly humans. J. Virol. 79, 5833-5838 (2005).

23. Donoghue, M. et al. A novel angiotensin-converting enzyme-related carboxypeptidase (ACE2) converts angiotensin I to angiotensin 1-9. Circ. Res. 87, E1-9 (2000).

24. Tipnis, S.R. et al. A human homolog of angiotensin-converting enzyme. Cloning and functional expression as a captopril-insensitive carboxypeptidase. J. Biol. Chem. 275, 33238-33243 (2000)

25. Corvol, P., Williams, T.A. \& Soubrier, F. Peptidyl dipeptidase A: angiotensin I-converting enzyme. Methods Enzymol. 248, 283-305 (1995).

26. Inagami, T. et al. Cloning, expression and regulation of angiotensin II receptors. Adv. Exp. Med. Biol. 377, 311-317 (1995).

27. Myint, S.H. Human coronaviruses-a brief review. Rev. Med. Virol. 4, 35-46 (1994).

28. Itoyama, S. et al. ACE1 polymorphism and progression of SARS. Biochem. Biophys. Res. Commun. 323, 1124-9 (2004).

29. Zou, K. et al. Analysis on the SARS-CoV genome of PUMCO1 isolate. Zhongguo $Y_{i}$ Xue Ke Xue Yuan Xue Bao 25, 495-498 (2003).

30. Imai, $Y$. et al. Comparison of lung protection strategies using conventional and high-frequency oscillatory ventilation. J. Appl. Physiol. 91, 1836-1844 (2001). 